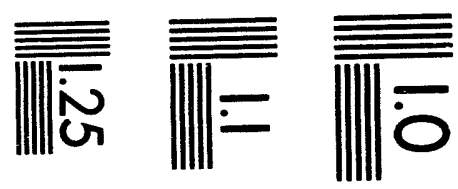

$$
\begin{aligned}
& \text { 秦背 }
\end{aligned}
$$

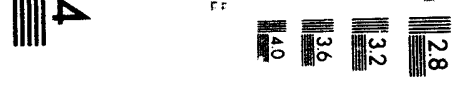

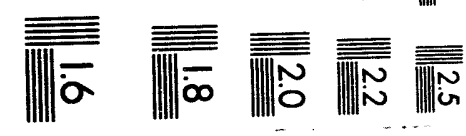



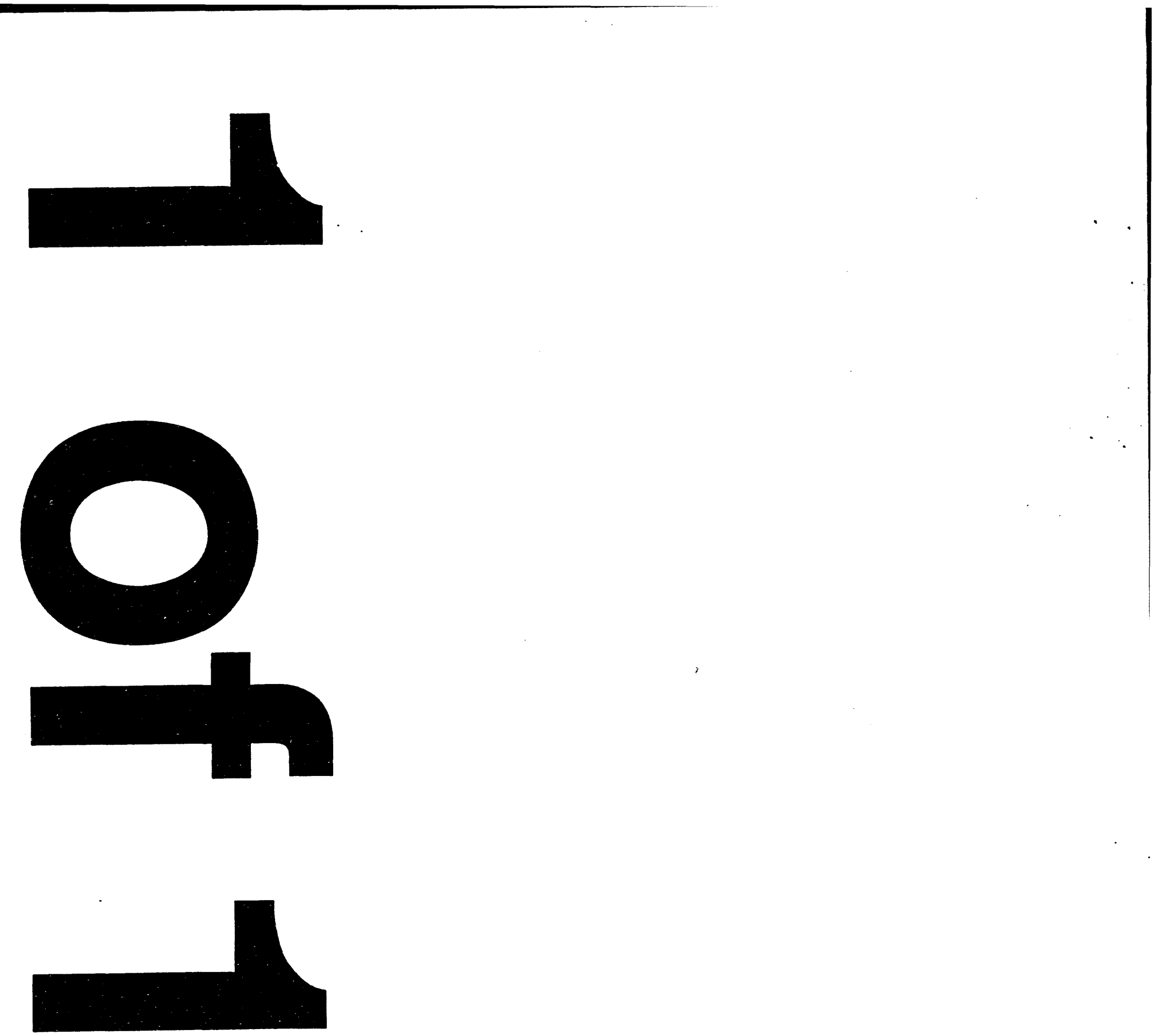


\section{DOEIER/143/5-T $\mid$}

PROGRESS REPORT

First Budget Period

15 December 1992 - 14 December 1993

and

RESEARCH PLAN

Second Budget Period

15 December 1993 -14 December 1994

DOE Grant No. DE-FG03-93ER14315

The Hydrophobic Character of Nonsulfide Mineral

Surfaces as Influenced by Double-Bond Reactions

of Adsorbed Unsaturated Collector Species

Submitted to: Clement R. Yonker

ER-14

Division of Chemical Sciences

Office of Basic Energy Science

Department of Energy

Washington DC 20585

Submitted by: J. D. Miller

Professor of Metallurgical Engineering

216 W. C. Browning Bldg.

University of Utah

Salt Lake City, UT 84112

(801) 581-5160

July 1993

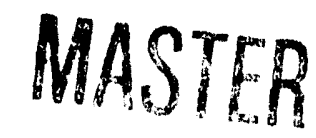

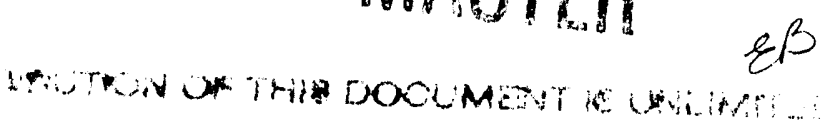




\section{Technical Progress during the First Budget Period \\ DOE Grant No. DE-FG03-93ER14315 \\ 15 December 1992 - 14 December 1993}

In the first budget period, different collector adsorption phenomena including adsorption density, adsorption state, and conformation have been examined for soluble salt, semi-soluble salt and insoluble oxide mineral systems. In the case of the soluble salt system, the influence of crystal lattice defects on the surface charge of $\mathrm{KCl}$ and it's flotation response was studied. In the case of semi-soluble salt minerals the behavior of fatty acid collectors adsorbed at the surfaces of calcite and fluorite was determined by in-situ Fourier transform infrared internal reflection

spectroscopy (FT-IR/IRS), multichannel laser Raman spectroscopy (MLRS), and LangmuirBlodgett (LB) techniques. Finally, changes in the hydrophobic character of the insoluble oxide mineral surfaces of sapphire and quartz were examined with respect to the aggregative nature of the adsorbed collector phase. A number of papers and presentations were prepared from this research and these contributions are listed at the end of this progress report.

\section{Soluble Salt Minerals}

During the previous grant period, nonequilibrium electrokinetic measurements using laserDoppler electrophoresis were reported for the first time for alkali halides and the sign of their surface charge thus determined. These new results are generally as had been expected from simplified lattice ion hydration theory with $\mathrm{KCl}$, under normal circumstances, being the most significant exception. In this regard, lattice defects have been found to influence the surface charge of $\mathrm{KCl}$ and it's flotation response. In the first budget period, the influence of crystal lattice defects ( $\mathrm{F}$ centers and oxygen imperfections) on the surface charge of $\mathrm{KCl}$ was studied 


\section{TABLE OF CONTENTS}

Technical Progress During the First Budget Period $\ldots \ldots \ldots \ldots \ldots \ldots \ldots$

Soluble Salt Minerals $\ldots \ldots \ldots \ldots \ldots \ldots \ldots \ldots \ldots \ldots \ldots \ldots$

Semi-Soluble Minerals $\ldots \ldots \ldots \ldots \ldots \ldots \ldots \ldots \ldots \ldots \ldots \ldots$

Insoluble Oxide Minerals $\ldots \ldots \ldots \ldots \ldots \ldots \ldots \ldots \ldots \ldots$

Publications and Presentations $\ldots \ldots \ldots \ldots \ldots \ldots \ldots \ldots \ldots$

Research Plans for the Second Budget Period $\ldots \ldots \ldots \ldots \ldots \ldots \ldots \ldots$ 
by varying the concentration of these defects in the crystal lattice of $\mathrm{KCl}$. In particular, the presence of oxygen defect states in the crystal lattice has been found to be responsible for $\mathrm{KCl}$ 's unexpected behavior based on the simplified lattice ion hydration theory.

The concentration of oxygen defect states in $\mathrm{KCl}$, as characterized by UV/Vis absorption, was varied in order to study their influence on the electrokinetic behavior and subsequently on the flotation response of $\mathrm{KCl}$. At low oxygen contents $(<60 \mathrm{ppm}) \mathrm{KCl}$ is positively charged as expected from lattice ion hydration theory. However, typically, $\mathrm{KCl}$ contains substantial oxygen ( $>300 \mathrm{ppm}$ ) sufficient to impart a negative surface charge which thus accounts for the selective flotation of $\mathrm{KCl}$ from $\mathrm{NaCl}$ by heterocoagulation with oppositely charged alkyl amine hydrochloride collector colloids.

In addition, the surface charge/collector colloid adsorption hypothesis has been extended to successfully explain the flotation behavior of certain alkali oxyanions including nitrates and sulfates as well as double salts including schoenite $\left(\mathrm{K}_{2} \mathrm{SO}_{4} \cdot \mathrm{MgSO}_{4} 6 \mathrm{H}_{2} \mathrm{O}\right)$ and kainite $\left(\mathrm{KCl} \cdot \mathrm{MgSO}_{4} \cdot 3 \mathrm{H}_{2} \mathrm{O}\right)$. As was done previously, the sign of the surface charge for these alkali oxyanions and double salts was established from both equilibrium and nonequilibrium electrokinetic measurements by laser-Doppler electrophoresis (Figure 1). These results together with the results from flotation/bubble attachment experiments indicate that the surface charge/collector colloid adsorption hypothesis accounts for the flotation response of many different soluble salt minerals when alkyl amines and carboxylates are used as collectors.

\section{Semi-Soluble Salt Minerals}

During the first budget period, the effect of fatty acid unsaturation on adsorption, polymerization, and subsequent hydrophobicity at a fluorite surface has been studied using the 


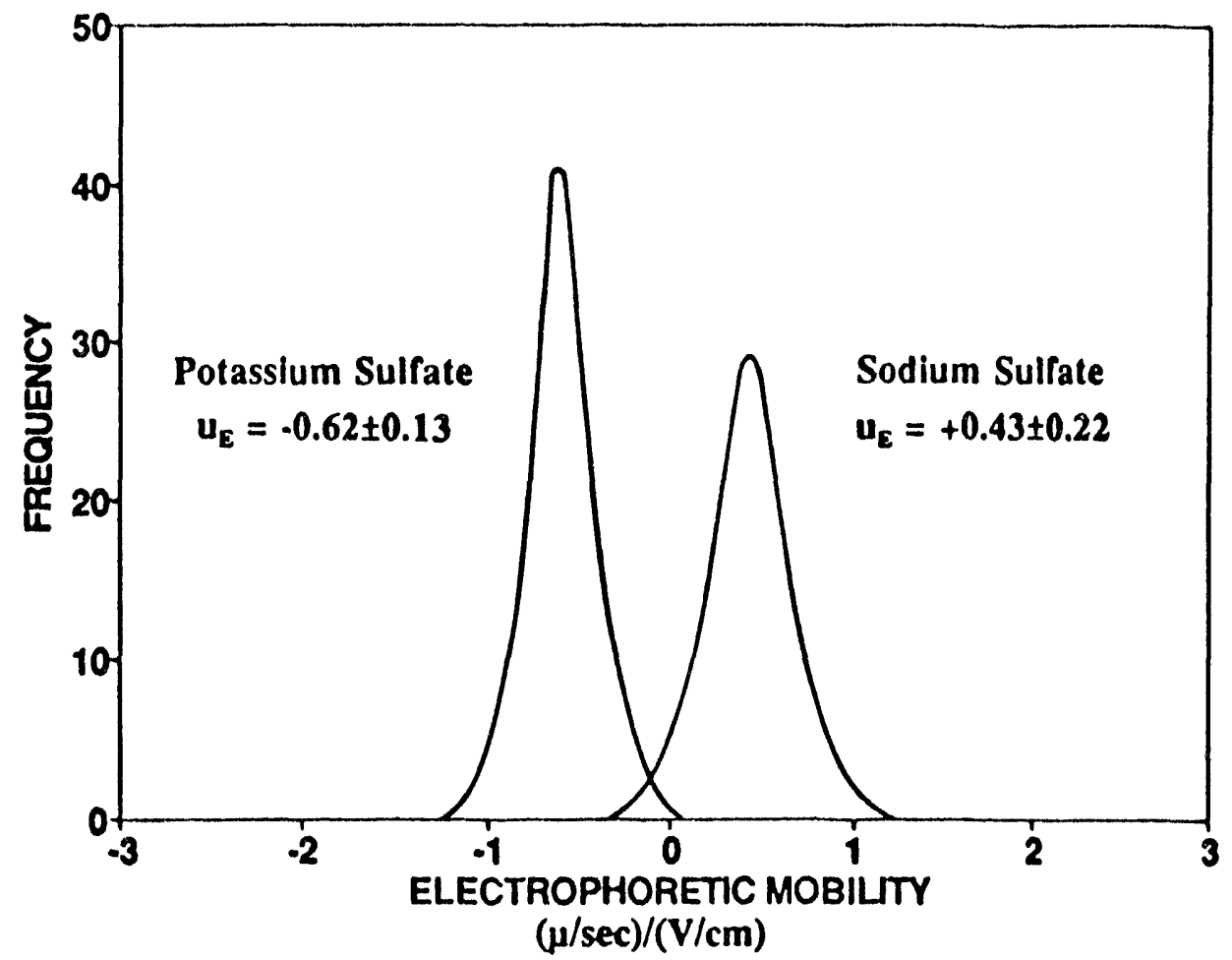

Figure 1. Nonequilibrium electrophoretic mobility distributions for potassium sulfate and sodium sulfate salts.

carboxylate anions of 18-carbon fatty acids. From in-situ FTIR/IRS experiments with a fluorite internal reflection element, it appears that for carboxylate anion concentrations less than $10^{-5} \mathrm{M}$, increasing the unsaturation increases the equilibrium adsorption density. For higher concentrations, the opposite relationship exists. In addition, it appears that polymerization via double bond oxidation occurs to such an extent with linoleate, which contains two double bonds, that the hydrophobicity as evidenced by contact angle measurements, decreases. Whereas with oleate, which contains only one double bond and apparently does not polymerize to the same extent, the hydrophobicity increases with polymerization.

Importantly, the validity of the FTIR/IRS adsorption density equation for quantitative analysis of surfactant adsorption has been confirmed by the study of transferred Langmuir- 
Blodgett (LB) films. In addition, a significant amount of time has been devoted to developing a clear understanding of the role of sample and incident beam areas on the use of FTIR/IRS adsorption density equation. Experimental conditions (beam areas) under which the FTIR/RS adsorption density equation can be used have been established.

The change of molecular orientation with respect to molecular area has been studied for transferred LB monolayer films of saturated and unsaturated fatty acids at the surf $\mathrm{e}$ of fluorite by FTIR/IRS. A monolayer with a more ordered structure (vertical orientation to the surface) was obtained for the transferred LB films of stearic acid than for transferred films of oleic acid, as is evident from the dichroic ratios plotted vs. adsorption density in Figure 2. In general, these results reveal the effect of unsaturation on the orientation of the hydrocarbon chain at a mineral surface.

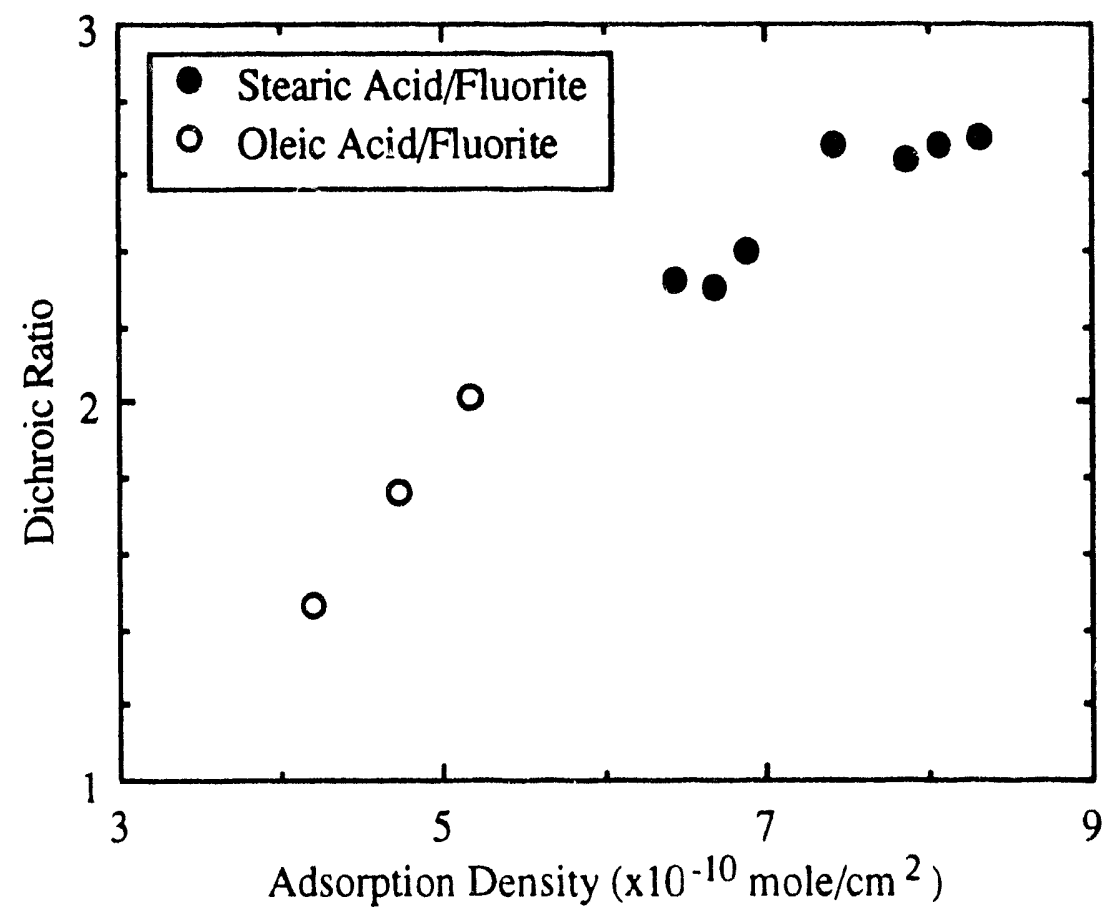

Figure 2. Dichroic ratio at the $\mathrm{CH}_{2}$ asymmetric stretching frequency vs. adsorption density of saturated and unsaturated fatty acid at the fluorite surfaces 
In-Situ FTIR/RS studies on fluorite and calcite indicated that the hydrophobicity, measured by contact angle, of the fluorite/oleate surface is greater than that of calcite for equivalent adsorption densities. The monolayer region has been confirmed to be chemisorbed oleate at both calcite and fluorite surfaces. Furthermore, the results from multichannel laser raman spectroscopy (MLRS) showed that chemisorbed oleate at the fluorite surface undergoes a doublebond reaction with oxygen to form an epoxide/polyether complex. Such a reaction, however, could not be detected at the calcite surface.

\section{Insoluble Oxide Minerals}

The hydrophobic character of sapphire surfaces in sodium dodecyl sulfate (SDS) solutions was examined with respect to the aggregative nature of the adsorbed surfactant phase as revealed in Table 1. It was found that, at equilibrium, sapphire surfaces having adsorbed SDS bilayers exhibited no contact angle. Conversely, when the adsorbed SDS appeared to be micellar in nature, the sapphire surfaces developed significant hydrophobic character with a well defined contact angle. The surface states as detected by in-situ FTIR/IRS conformational analysis were found to be reversible with temperature.

For the quartz/dodecylamine system, no surface phase changes of the adsorbed surfactant were observed. This behavior was expected from examination of the phase behavior of dodecylamine in solution. Evidence was seen for the adsorption of colloidal precipitates of dodecylamine at the quartz surface at $\mathrm{pD} 8.2$ but not at $\mathrm{pD} 6.0$. 
Table 1. Hydrophobic Character of Sapphire Surfaces in Sodium Dodecyl Sulfate Solutions at pH 2.85 and $298 \mathrm{~K}$

\begin{tabular}{|c|c|c|c|}
\hline $\begin{array}{c}\text { Concentration } \\
M\end{array}$ & Phase & $\begin{array}{c}\text { Contact Angle, } \\
\text { degrees }\end{array}$ & $\begin{array}{c}\text { Adsorption Density, } \\
\text { mol/cm }\end{array}$ \\
\hline $1.7 \times 10^{-5}$ & cyl. mic. & NA & $5 \times 10^{-11}$ \\
\hline $4.5 \times 10^{-5}$ & cyl. mic. & $43.4 \pm 2.7$ & $8 \times 10^{-11}$ \\
\hline $6.6 \times 10^{-5}$ & cyl. mic. & $42.1 \pm 1.6$ & $1 \times 10^{-10}$ \\
\hline $2.5 \times 10^{-4}$ & cyl, mic. & $34.4 \pm 2.1$ & $2 \times 10^{-10}$ \\
\hline $3.0 \times 10^{-4}$ & coa / cyl. mic. & $34.5 \pm 1.0$ & $3 \times 10^{-10}$ \\
\hline $4.4 \times 10^{-4}$ & coa & NA & $5 \times 10^{-10}$ \\
\hline $1.2 \times 10^{-3}$ & coagel & NA & $1 \times 10^{-9}$ \\
\hline $1.5 \times 10^{-3}$ & coagel & NA & $1 \times 10^{-9}$ \\
\hline $3.2 \times 10^{-3}$ & coagel & NA & $1 \times 10^{-9}$ \\
\hline
\end{tabular}

where NA = no attachment; cyl. mic. = cylindrical micelle; coa = coagel.

\section{Publications and Presentations}

1. M. R. Yalamanchili, and J. D. Miller, The Surface Charge of $\mathrm{KCl}$ as Influenced by Crystal Lattice Defects, Submitted to the Journal of Colloid and Interface Science, 1993.

2. J. D. Miller, and M. R. Yalamanchili, Fundamental Aspects of Soluble Salt Flotation, to be presented at the Minerals Engineering '93 conference, August 25-27, Cape Town, South Africa, 1993.

3. M. L. Free, and J. D. Miller, The Effect of Sample Area in Adsorption Density Calculations from FT-IR/IRS Experiments, to be submitted to the Journal of Applied Spectroscopy, 1993. 
4. W. H. Jang, and J. D. Miller, Verification of the IRS Adsorption Density Equation by FTIR Analysis of Transferred Langmuir-Blodgett Films, Langmuir, in press, 1993.

5. C. A. Young, K. Knutson, and J. D. Miller, Significance of Temperature Control in FTNIR Spectrometers, Applied Spectroscopy, 47, 1, pp. 7-11, 1993.

6. C. A. Young, and J. D. Miller, Comparison of Oleate Adsorption at Calcite and Fluorite Surfaces: An In-Situ FT-NIR/IRS and MLRS Study, Presented at the 122nd SME Annual Meeting, Feb. 15-18, Reno, NV, Preprint \# 93-224, 1993,

7. W. M. Cross, and J. D. Miller, Adsorbed Surfactant Hydrocarbon Chain Conformation and its Effect on Hydrophobicity, Presented at the 122nd SME Annual Meeting, Feb. 15-18, Reno, NV, Preprint \# 93-213, 1993.

8. C. A. Young, and J. D. Miller, Effect of Temperature on Oleate Adsorption at a Calcite Surface, Part I : FT-IR/IRS Study and Review, Submitted to Colloids and Surfaces, 1993. 


\section{RESEARCH PLANS FOR THE SECOND BUDGET PERIOD}

Excellent progress has been made during first budget period as indicated by the number of publications and presentations listed in the progress report. These efforts are expected to continue throughout the next budget period. An experienced research team is in place and the intended research activities in each of the three mineral systems are described below.

\section{Soluble Salt Minerals}

The proposed surface charge/collector colloid adsorption hypothesis suggests that a heterocoagulation event occurring between oppositely charged collector colloids and the salt mineral particles is responsible for salt mineral flotation. A more detailed investigation will be undertaken during the second budget period to study (1) the physical characteristics of the adsorbing collector colloids at the salt surfaces and (2) the interaction forces responsible for the heterocoagulation event between the oppositely charged particles in order to further substantiate the proposed adsorption mechanism. These studies will be done using techniques such as photon correlation spectroscopy and atomic force microscopy. Efforts are underway to purchase our own atomic force microscope.

In addition, hydration and water structure at salt surfaces in saturated brines will be studied by the examination of -OD stretching bands of the in-situ FTIR/IRS spectra. These results will be compared to the $\mathrm{D}_{2} \mathrm{O}$ spectra obtained at the surfaces of germanium, sapphire, and rutile at high ionic strengths in order to study the influence of surface charge and ionic strength on surface hydration phenomena. It is intended to determine if the water structure at the mineral surface can be used to explain important heterocoagulation phenomena that have been observed in this system. 


\section{Semi-Soluble Minerals}

It is anticipated that during the next budget period, considerable effort will be focussed upon a continuation of the research to determine the effect of the degree of unsaturation on adsorption phenomena. In addition, considerable effort will be directed towards understanding the effect of molecular orientation on surface hydrophobicity and polymerization reactions. A large amount of spectral data from both self-assembly and transferred Langmuir-Blodgett monolayers will be compared to gain insight into the structure of the adsorbed molecules. This data will be combined with contact angle and bubble attachment time measurements for both polymerized and nonpolymerized layers to determine the effect of orientation on polymerization and hydrophobicity.

Also, the relationship between the extent of polymerization and hydrophobicity will be characterized using $\mathrm{SrF}_{2}$ and $\mathrm{CaF}_{2}$ sputter-coated $\mathrm{SrF}_{2}$ internal reflection elements which are transparent in the $1090 \mathrm{~cm}^{-1}$ region where C-O-C band appears upon polymerization. A correlation will be obtained by comparing the $1090 \mathrm{~cm}^{-1}$ absorbance values with the resulting hydrophobicity as evidenced by contact angle and contact time measurements.

The molecular orientation of LB surfactant monolayers and the polymerization of transferred LB monolayer films of unsaturated surfactants will be investigated at the fluorite, and germanium surfaces. The effect of electrical stimulation of the substrate on molecular orientation and polymerization of both self assembly and transferred LB films will be investigated by in-situ spectroscopic examination of the IRE under electrochemical control. 


\section{Insoluble Oxide Minerals}

In the coming year, the adsorption behavior of unsaturated collectors on alumina will be determined and the hydrophobicity evaluated using in-situ FTIR/IRS and transferred LB films. In addition, the effect of fatty acid unsaturation on adsorption, polymerization, and subsequent hydrophobicity at a rutile surface will be studied using in-situ FTIR/IRS and transferred LB films. Efforts will be made to overcome the birefringence problems associated with the use of a rutile IRE. 

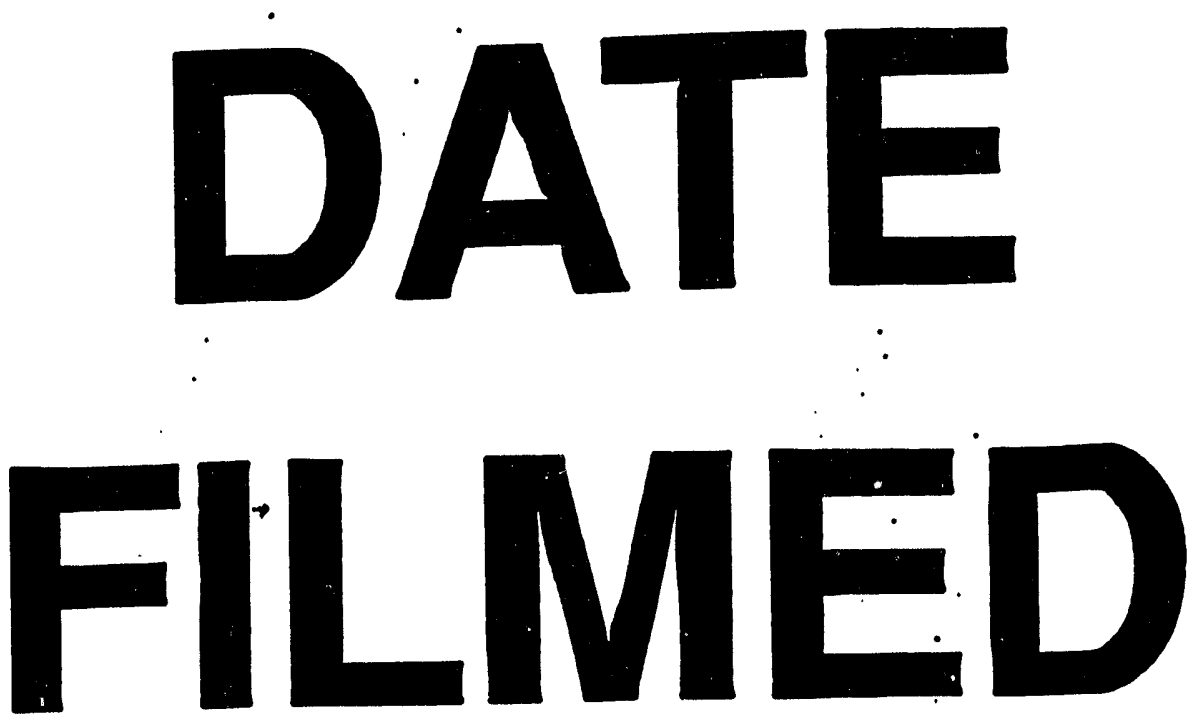

$4 / 11 / 94$
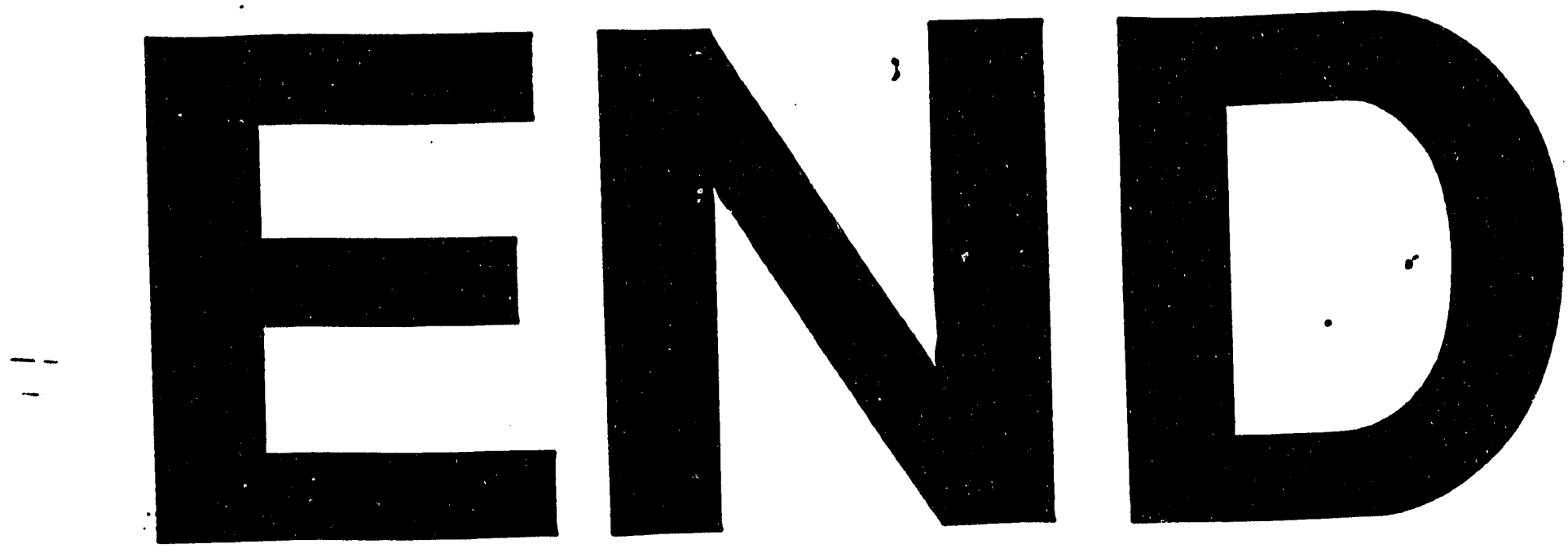

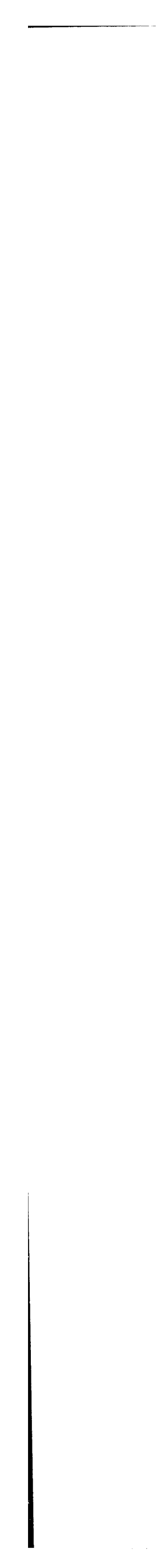